This document is published in:

European Journal of Mechanics B/Fluids, Vol. Nov.-Dec., no 36, pp. 128-138

DOI: http://dx.doi.org/10.1016/j.euromechflu.2012.03.016

(C) 2012 Elsevier Masson SAS. 


\title{
Viscous stability analysis of jets with discontinuous base profiles
}

\author{
W. Coenen*, A. Sevilla, A.L. Sánchez \\ Área de Mecánica de Fluidos, Universidad Carlos III de Madrid, Av. Universidad 30, 28911 Leganés, Spain
}

Keywords:

Hydrodynamic stability

Jets

Discontinuous profiles

Jump conditions

\begin{abstract}
A B S T R A C T
The viscous linear stability of parallel gaseous jets with piecewise constant base profiles is considered in the limit of low Mach numbers. Our results generalise those of Drazin [P.G. Drazin, Discontinuous velocity profiles for the Orr-Sommerfeld equation J. Fluid Mech. 10 (1961) 571-583], by contemplating the possibility of arbitrary jumps in density and transport properties between two uniform streams separated by a vortex sheet. The eigenfunctions, obtained analytically in the regions of uniform flow, are matched through an appropriate set of jump conditions at the discontinuity of the basic flow, which are derived by repeated integration of the linearised conservation equations in their primitive variable form. The development leads to an algebraic dispersion relation of ample validity that explicitly accounts for the parametric dependence of the stability properties on the jet-to-ambient density ratio, the Reynolds number, the Prandtl number, and the exponent of the presumed power-law dependence of viscosity and thermal conductivity on temperature. The dispersion relation is validated through comparisons with stability calculations performed with continuous profiles and is applied, in particular, to study the effects of molecular transport on the spatiotemporal stability of parallel non-isothermal gaseous jets with very thin shear layers. The eigenvalue computations performed by using the vortex-sheet model are shown to be several orders of magnitude faster than those associated with continuous profiles with thin shear layers.
\end{abstract}

\section{Introduction}

Many of the early efforts to investigate the instability of parallel shear flows were based on simple flow configurations with discontinuous base velocity profiles, with prominent examples being the inviscid vortex-sheet investigations of Helmholtz [1] and Kelvin [2], and Rayleigh's stability analysis of the shear layer with piecewise linear velocity profile [3]. These inviscid analyses involve integration of Rayleigh's equation for the perturbations of the stream function with appropriate matching conditions at the discontinuities, corresponding to the requirement that the pressure and the normal velocity be continuous across the material interface. Viscous effects can be taken into account in the perturbations through the use of the Orr-Sommerfeld equation, as done by Esch [4] for the analysis of Rayleigh's piecewise linear configuration and by Drazin [5] for base flows with discontinuous velocity profiles. The latter showed that the correct set of viscous jump conditions at the discontinuity of the base velocity profile can be derived by sequentially integrating the Orr-Sommerfeld equation four times across the discontinuity, and then taking the limit of vanishing integration intervals in each one of the four expressions obtained.

\footnotetext{
* Corresponding author. Tel.: +34 916248344.

E-mail address: wcoenen@ing.uc3m.es (W. Coenen).
}

As discussed by Drazin [5], the use of discontinuous base profiles as a leading-order representation for the flow field, with the shear layers being substituted by vortex sheets, is a valid approach for the analysis of normal modes with wavelength much larger than the shear-layer thickness. The latter condition also ensures that there is no contradiction in retaining viscous effects in the perturbations while neglecting them in the basic flow [5].

The advantage of Drazin's approach is that it leads to a closed algebraic expression for the dispersion relation, thereby facilitating analytical developments and reducing computational costs. The corresponding dispersion relations for the plane mixing layer and the symmetric plane jet, obtained by Drazin [5], were shown to be in agreement with the corresponding lowwavenumber approximations for continuous base profiles, which had been previously obtained by Tatsumi [6] and by Tatsumi [7], respectively, thus demonstrating the validity of the approach. It is important to emphasise that, as noticed by Drazin [5], in the limit $\operatorname{Re} \rightarrow \infty$, the viscous dispersion relation obtained with the discontinuous approximation does not reduce to the inviscid one, derived from Rayleigh's [3] equation. This singular behaviour can be explained by the fact that the former case corresponds to taking the low-wavenumber limit $k \rightarrow 0$ for fixed $\operatorname{Re} / k$, and subsequently letting $\operatorname{Re} \rightarrow \infty$, while the latter case is based on the limit $k \operatorname{Re} \rightarrow \infty$.

An important example satisfying the long wavelength condition mentioned above are the columnar modes of jets, which are 
known to play a relevant role in their stability [8], and dominate their dynamics when self-excited by becoming locally absolutely unstable in the near field [9-13]. Previous viscous stability analyses of these jet flows, including planar [12] and axisymmetric [14] configurations, have made use of model base profiles with thin shear layers. Since the relevant wavelengths for the columnar modes are of the order of the jet radius, the shear-layer thickness enters in the analysis as a secondary parameter that takes small non-zero values. The relevant limit of vanishing shear-layer thickness is difficult to investigate numerically with continuous base profiles, because the associated integrations become very costly when the shear-layer thickness takes small values. Consideration of discontinuous base profiles arises as a natural alternative in this case and serves as motivation for the present work.

In a subsequent study, Drazin [15] extended his model to include the effects of variable density and viscosity, but only for incompressible flow and excluding heat conduction and species diffusion. Therefore, the results obtained by Drazin $[5,15]$ exclude many flows of practical relevance. This fact motivates the present study, whose main objective is to provide a framework that, although based on the ideas originally proposed by Drazin $[5,15]$, extends his results to contemplate axisymmetric non-buoyant gaseous jet flows with small values of the Mach number. The required development involves an extension of Drazin's work to cylindrical geometries and non-solenoidal disturbances. The analysis needs to employ the linear stability equations in their primitive variable form. Repeated integrals of these equations provide the necessary jump conditions for the perturbations across the discontinuities of the base profiles.

Specifically, the present work provides the following extensions to Drazin's approach: first, while only two-dimensional base flows are treated by Drazin [5,15], the present study considers axisymmetric base flows, contemplating disturbances of arbitrary azimuthal wavenumber. In addition, in contrast with the works of Drazin $[5,15]$, our approach includes heat conduction, as well as the dependence of viscosity and thermal conductivity on temperature. Although constant molecular weight is employed in the development, so that density changes are only associated with temperature changes, extension of the results to variable molecular weight can be readily made, as indicated in the text.

The paper is organised as follows. The formulation of the linear stability problem is presented in Section 2, followed in Section 3 by the discontinuous formalism developed for the limit of vanishingly thin shear layers, including the eigenfunctions and jump conditions that must be used to obtain the algebraic dispersion relation for the particular case of non-isothermal jets of ideal gases with a presumed power-law temperature dependence for the viscosity and thermal conductivity. The validity of the dispersion relation is assessed in Section 4 by comparisons with the results obtained by integrating numerically the linearised stability equations with continuous base profiles in the limit of very thin shear layers. The dispersion relation is utilised to study the spatiotemporal stability of axisymmetric jets, obtaining, in particular, the dependence on molecular transport of the critical conditions for transition between convective and absolute instabilities. Finally, Section 5 is devoted to conclusions.

\section{Stability equations}

We consider the linear stability of a gas jet discharging with a constant flow rate into an ambient atmosphere of the same fluid. The characteristic jet velocity, given for instance by its centreline value $U_{0}^{*}$, is assumed to be much smaller than the ambient velocity of sound $c_{\infty}^{*}$, so that the simplifications associated with the low-Mach-number approximation, commonly used to analyse low-speed reactive flows [16,17], apply to the description of the resulting jet flow. In particular, in low-Mach-number flows, the spatial pressure variations, of the order of the characteristic dynamic pressure, are a factor $\left(U_{0}^{*} / c_{\infty}^{*}\right)^{2}$ smaller than the ambient pressure $p_{\infty}^{*}$, and can be therefore neglected when writing the equation of state for the gas

$\frac{p_{\infty}^{*}}{\rho^{*}}=\frac{R^{o}}{W^{*}} T^{*}$,

where $R^{o}$ is the universal gas constant and $W^{*}$ denotes the mean molecular weight of the gas mixture, with the asterisk * used in the formulation to denote dimensional magnitudes. As can be seen above, although the flow is incompressible, in the sense that the pressure variations are small, significant density variations may appear associated with either temperature or molecular-weight variations.

Simplifications can be introduced also in writing the energy balance, because the relative enthalpy variations induced by spatial changes of pressure and also those induced by viscous dissipation are of order $\left(U_{0}^{*} / c_{\infty}^{*}\right)^{2}$, and can be therefore neglected in the first approximation when describing jet flows with $U_{0}^{*} / c_{\infty}^{*} \ll 1$, for which changes in enthalpy are only due to heat conduction.

To formulate the problem, the velocity $\boldsymbol{u}^{*}$, density $\rho^{*}$, temperature $T^{*}$, shear viscosity $\mu^{*}$, and thermal conductivity $\kappa^{*}$ are scaled with their characteristic centreline values $U_{0}^{*}, \rho_{0}^{*}, T_{0}^{*}, \mu_{0}^{*}$, and $\kappa_{0}^{*}$, while the bulk viscosity $\mu_{V}^{*}$ is scaled with $\mu_{0}^{*}$. The pressure variation from the reference value $p_{\infty}^{*}$ is scaled with the characteristic dynamic pressure $\rho_{0}^{*} U_{0}^{* 2}$, as corresponds to the lowMach-number approximation considered here. The jet radius $a^{*}$ is used to give the dimensionless cylindrical coordinates $(x, r, \theta)$, whereas the time $t$ is scaled with $a^{*} / U_{0}^{*}$. The unit vectors of the coordinate system are represented by $\left(\boldsymbol{e}_{\boldsymbol{x}}, \boldsymbol{e}_{\boldsymbol{r}}, \boldsymbol{e}_{\boldsymbol{\theta}}\right)$.

The stability analysis begins by assuming that the flow can be decomposed into a steady parallel basic flow in the $x$ direction and small fluctuating disturbances according to $\boldsymbol{u}^{*} / U_{0}^{*}=\boldsymbol{U}+$ $\boldsymbol{u}, \rho^{*} / \rho_{0}^{*}=\bar{\rho}+\rho, T^{*} / T_{0}^{*}=\bar{T}+T, \mu^{*} / \mu_{0}^{*}=\bar{\mu}+\mu, \mu_{V}^{*} / \mu_{0}^{*}=$ $\bar{\mu}_{V}+\mu_{V}, \kappa^{*} / \kappa_{0}^{*}=\bar{\kappa}+\kappa$ and $p^{*} /\left(\rho_{0}^{*} U_{0}^{* 2}\right)=p$, with $\boldsymbol{u}=(u, v, w)$. The base-flow variables, which, with the exception of the velocity $\boldsymbol{U}=(U, 0,0)$, are denoted by a bar, are allowed to vary with the transverse coordinate $r$, except for the base pressure variation, which is identically zero in the parallel flow approximation used here.

Following the usual methods of hydrodynamic stability, the continuity, momentum and energy conservation equations are linearised around the base flow, yielding the following system of linear equations for the disturbances

$$
\begin{aligned}
& \frac{\partial \rho}{\partial t}+U \frac{\partial \rho}{\partial x}+\bar{\rho} \nabla \cdot \boldsymbol{u}+\bar{\rho}^{\prime} v=0 \\
& \bar{\rho}\left(\frac{\partial \boldsymbol{u}}{\partial t}+U \frac{\partial \boldsymbol{u}}{\partial x}+U^{\prime} v \boldsymbol{e}_{\boldsymbol{x}}\right) \\
& =-\nabla p+\frac{1}{\operatorname{Re}}\left[\bar{\mu} \nabla^{2} \boldsymbol{u}+\left(\bar{\mu}_{V}+\frac{1}{3} \bar{\mu}\right) \nabla(\nabla \cdot \boldsymbol{u})\right. \\
& \quad+\left(\bar{\mu}_{V}^{\prime}-\frac{2}{3} \bar{\mu}^{\prime}\right)(\nabla \cdot \boldsymbol{u}) \boldsymbol{e}_{\boldsymbol{r}}+\bar{\mu}^{\prime}\left(\nabla \boldsymbol{u}+\nabla \boldsymbol{u}^{\mathrm{T}}\right) \cdot \boldsymbol{e}_{\boldsymbol{r}} \\
& \left.\quad+\left(U^{\prime} \mu^{\prime} \boldsymbol{e}_{\boldsymbol{x}}+U^{\prime} \frac{\partial \mu}{\partial x} \boldsymbol{e}_{\boldsymbol{r}}\right)+\left(U^{\prime \prime}+\frac{U^{\prime}}{r}\right) \mu \boldsymbol{e}_{\boldsymbol{x}}\right], \\
& \bar{\rho}\left(\frac{\partial T}{\partial t}+U_{\frac{\partial T}{\partial x}}+\bar{T}^{\prime} v\right) \\
& \quad \frac{1}{\operatorname{Re} \operatorname{Pr}}\left[\bar{\kappa} \nabla^{2} T+\bar{\kappa}^{\prime} T^{\prime}+\bar{T}^{\prime} \kappa^{\prime}+\left(\bar{T}^{\prime \prime}+\frac{\bar{T}^{\prime}}{r}\right) \kappa\right],
\end{aligned}
$$


where the prime' denotes differentiation with respect to $r$ and $\nabla$ is the dimensionless nabla operator. In the formulation, $\operatorname{Re}=$ $\rho_{0}^{*} U_{0}^{*} a^{*} / \mu_{0}^{*}$ and $\operatorname{Pr}=c_{p}^{*} \mu_{0}^{*} / \kappa_{0}^{*}$ are the Reynolds and Prandtl numbers, with $c_{p}^{*}$ representing the specific heat at constant pressure for the gas mixture, assumed to be constant. The effect of body forces has been neglected in (2b). Also, both compressibility and viscous dissipation have been discarded in (2c), an approximation that, as explained before, involves relative errors of the order of the square of the Mach number, negligibly small in the limit of low Mach numbers considered here.

The following analysis focuses on wave-like perturbations of the form $\left(\rho, T, \mu, \mu_{V}, \kappa, u, v, w, p\right)=\left(\hat{\rho}, \hat{T}, \hat{\mu}, \hat{\mu}_{V}, \hat{u}, \mathrm{i} \hat{v}, \hat{w}, \hat{p}\right)$ $\exp [i(k x+m \theta-\omega t)]$ with $k$ and $m$ denoting the streamwise and azimuthal wave numbers and $\omega$ being the angular frequency. Substituting these normal modes into $(2 \mathrm{a})-(2 \mathrm{c})$ then yields the set of stability equations that will form the starting point for the discontinuous formalism in the remainder of this work:

$$
\begin{aligned}
& (k U-\omega) \hat{\rho}+k \bar{\rho} \hat{u}+\frac{(r \bar{\rho} \hat{v})^{\prime}}{r}+\frac{m \bar{\rho} \hat{w}}{r}=0, \\
& \bar{\mu} \hat{\nabla}_{\beta, m}^{2} \hat{u}-\mathrm{iRe} U^{\prime} \bar{\rho} \hat{v}-\mathrm{iRe} k \hat{p}-\left(\bar{\mu}_{V}+\frac{\bar{\mu}}{3}\right) k \\
& \quad \times\left(k \hat{u}+\frac{(r \hat{v})^{\prime}}{r}+\frac{m \hat{w}}{r}\right) \\
& \quad+\bar{\mu}^{\prime}\left(\hat{u}^{\prime}-k \hat{v}\right)+U^{\prime} \hat{\mu}^{\prime}+\frac{\left(r U^{\prime}\right)^{\prime} \hat{\mu}}{r}=0, \\
& \bar{\mu}\left(\hat{\nabla}_{\beta, m}^{2} \hat{v}-\frac{\hat{v}+2 m \hat{w}}{r^{2}}\right)+\mathrm{iRe} \hat{p}^{\prime}+\left(\bar{\mu}_{V}+\frac{\bar{\mu}}{3}\right) \\
& \quad \times\left[k \hat{u}^{\prime}+\left(\frac{(r \hat{v})^{\prime}}{r}\right)^{\prime}+m\left(\frac{\hat{w}}{r}\right)^{\prime}\right]+\left(\bar{\mu}_{V}^{\prime}-\frac{2 \bar{\mu}^{\prime}}{3}\right) \\
& \quad \times\left(k \hat{u}+\frac{(r \hat{v})^{\prime}}{r}+\frac{m \hat{w}}{r}\right)+2 \bar{\mu}^{\prime} \hat{v}^{\prime}+k U^{\prime} \hat{\mu}=0, \\
& \bar{\mu}\left(\hat{\nabla}_{\beta, m}^{2} \hat{w}-\frac{\hat{w}+2 m \hat{v}}{r^{2}}\right)-\frac{\mathrm{iRe} m \hat{p}}{r} \\
& \quad-\left(\bar{\mu}_{V}+\frac{\bar{\mu}}{3}\right) \frac{m}{r}\left(k \hat{u}+\frac{(r \hat{v})^{\prime}}{r}+\frac{m \hat{w}}{r}\right) \\
& \quad+\bar{\mu}^{\prime}\left[r\left(\frac{\hat{w}}{r}\right)^{\prime}-\frac{m \hat{v}}{r}\right]=0,
\end{aligned}
$$

$\bar{\kappa} \hat{\nabla}_{\gamma, m}^{2} \hat{T}-\operatorname{iRe} \operatorname{Pr} \bar{\rho} \bar{T}^{\prime} \hat{v}+\bar{\kappa}^{\prime} \hat{T}^{\prime}+\bar{T}^{\prime} \hat{\kappa}^{\prime}+\frac{\left(r \bar{T}^{\prime}\right)^{\prime} \hat{\kappa}}{r}=0$,

where the reduced Laplacian operator

$\hat{\nabla}_{\phi, m}^{2}=\frac{1}{r} \frac{\mathrm{d}}{\mathrm{d} r}\left(r \frac{\mathrm{d}}{\mathrm{d} r}\right)-\left(\phi^{2}+\frac{m^{2}}{r^{2}}\right)$

has been introduced for convenience. The quantity $\phi$ takes the values $\phi=\beta$ and $\phi=\gamma$ in the momentum and energy equations, respectively, with

$$
\begin{aligned}
& \beta=\left[k^{2}+i \operatorname{Re}(k U-\omega) \bar{\rho} / \bar{\mu}\right]^{1 / 2}, \\
& \gamma=\left[k^{2}+i \operatorname{Re} \operatorname{Pr}(k U-\omega) \bar{\rho} / \bar{\kappa}\right]^{1 / 2},
\end{aligned}
$$

defined with a non-negative real part for definiteness.

The above equations are to be supplemented with the equation of state (1) written in the low-Mach-number approximation, and with appropriate constitutive laws for the variation of the transport properties. In principle, when the flow involves a mixture of different gases, the composition enters in determining the mean molecular weight and the transport coefficients, so that the above equations should be supplemented with species conservation equations written for the mass fractions $\bar{Y}_{\alpha}+Y_{\alpha}$ of each different chemical species $\alpha$. The resulting equations for the perturbations $Y_{\alpha}$ would be similar to (3e), with the Schmidt number of each species replacing the Prandtl number and with the product of the density times the species diffusivity replacing the thermal conductivity. For simplicity in the development, variations of molecular weight will be neglected in the equation of state (1), which reduces to

$\bar{\rho}=1 / \bar{T}$

for the base flow. Also, presumed power laws

$\bar{\mu}=\bar{\kappa}=\bar{T}^{\sigma}$

will be introduced for the temperature variation of transport properties, so that the descriptions of the composition field and its disturbances will not be necessary. Nevertheless, comments on the extensions of the analysis to account for changes in composition are offered below in Section 5 .

The stability equations must be accompanied with suitable boundary conditions. In the far field, all perturbations must vanish,

$\left(\hat{\rho}, \hat{T}, \hat{\mu}, \hat{\mu}_{V}, \hat{\kappa}, \hat{u}, \hat{v}, \hat{w}, \hat{p}\right) \rightarrow 0 \quad$ as $r \rightarrow \infty$,

while at the centreline $r=0$ we have to impose a vanishing azimuthal dependence of the perturbations as the axis $r=$ 0 is approached [18], i.e. $\lim _{r \rightarrow 0} \partial\left(\rho, T, \mu, \mu_{V}, \kappa, u \boldsymbol{e}_{\boldsymbol{x}}+v \boldsymbol{e}_{\boldsymbol{r}}+\right.$ $\left.w \boldsymbol{e}_{\theta}, p\right) / \partial \theta=0$. This leads to boundary conditions at the axis that take the form

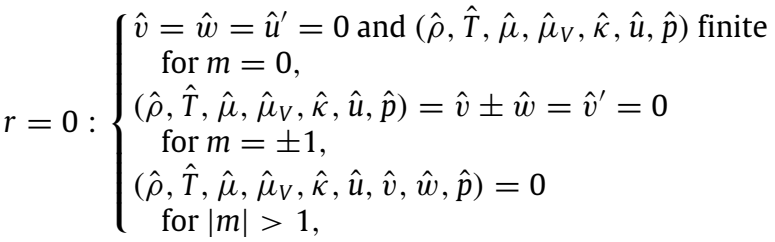

for different azimuthal modes.

The local stability properties of the parallel jet flow can now be obtained by solving the generalised eigenvalue problem set up by the system of ordinary differential equations (3a)-(3e) with boundary conditions (9)-(10). Hereby, eigenfunctions $(\hat{\rho}, \hat{T}$, $\left.\hat{\mu}, \hat{\mu}_{V}, \hat{\kappa}, \hat{u}, \hat{v}, \hat{w}, \hat{p}\right)$ only exist if $k$ and $\omega$ satisfy a dispersion relation

$D(k, \omega ; R)=0$

where $R$ indicates the set of control parameters that govern the flow.

If functional forms are specified for the base velocity and density profiles $U(r)$ and $\rho(r)$, the stability problem can be solved numerically. For instance, in the case of jets without ambient coflow, the hyperbolic tangent base velocity profile

$U(r)=\frac{1}{2}+\frac{1}{2} \tanh \left[\frac{1}{4 \theta}\left(\frac{1}{r}-r\right)\right]$,

is commonly used $[19,20,14,21-23]$, together with CroccoBusemann's relation,

$\bar{\rho}(r)=[S+(1-S) U]^{-1}$.

As can be seen in (12) and (13), the resulting base profiles depend on two parameters, namely the jet-to-ambient density ratio, $S$, and the momentum thickness, $\theta=\int_{0}^{\infty} U(1-U) \mathrm{d} r$.

The numerical computation of the jet stability problem is typically carried out with a spectral collocation method (for example $[24,14])$. To precisely determine the eigenvalues and eigenfunctions of the stability problem, the spatial distribution of the collocation points must be adequate to resolve simultaneously 
scales of the order of the jet radius, as well as small scales of the order of the shear-layer thickness. This means a minimum number of collocation points must be placed within the shear layer. For example, to obtain the numerical results to be presented in Section 4, we guaranteed 10 points to lie in the shear layer. Consequently, as the shear layer becomes thinner, the numerical computation demands an increasing number of Chebyshev collocation points. The resulting eigenvalue calculations become very costly, yielding computational times that are impractically long, even with modern computer systems. To facilitate the computation, it is therefore of interest to consider the limiting solution associated with shear layers of infinitesimally small thickness, when the base profiles become piecewise uniform, and the shear layer is substituted by a vortex sheet, the case investigated in the following section.

Note that the two parameters $S$ and $\theta$ are characteristic measures of the jump in density between the jet and the ambient and the thickness $\delta^{*}$ of the transition layer through which this jump takes place. Clearly, these two parameters would also uniquely define the base flow for other choices of the functional forms $U(r), \bar{\rho}(r)$. Although the hyperbolic tangent profiles (12)-(13) will be used in validations to illustrate the virtue of the discontinuous formalism to be introduced in the next section, the deduction of the formalism itself does not rely on this particular choice.

\section{Solution procedure for infinitesimally thin shear layers}

In this section, we consider the limiting solution of the eigenvalue problem (3a)-(3e), (9)-(10) associated with shear layers of infinitesimally small thickness, when the base profiles are piecewise uniform, with $U=\bar{\rho}=\bar{\mu}=\bar{\kappa}=1$ and $\bar{\mu}_{V}=\mu_{V-}$ for $0 \leq r<1$ and $U=0, \bar{\rho}=1 / S, \bar{\mu}=M, \bar{\kappa}=K$ and $\bar{\mu}_{V}=\bar{\mu}_{V+}$ for $r>1$. It is shown in Appendix A how for $0 \leq r<1$, the corresponding form of the stability equations (3a)-(3e) with boundary conditions (10) at the axis can be solved to give

$\hat{\rho}=A I_{m}\left(\gamma_{-} r\right)$,

$\hat{p}=C I_{m}(k r)-\frac{A \mathrm{i}(k-\omega)}{\operatorname{Re}}\left(\mu_{V-}+\frac{4}{3}-\frac{1}{\operatorname{Pr}}\right) I_{m}\left(\gamma_{-} r\right)$,

$\hat{u}=E I_{m}\left(\beta_{-} r\right)-\frac{C k}{k-\omega} I_{m}(k r)-\frac{A \mathrm{i} k}{\operatorname{Re} \operatorname{Pr}} I_{m}\left(\gamma_{-} r\right)$,

$\hat{v}=\hat{w}+G I_{m-1}\left(\beta_{-} r\right)+\frac{C k}{k-\omega} I_{m-1}(k r)+\frac{A \mathrm{i} \gamma_{-}}{\operatorname{Re} \operatorname{Pr}} I_{m-1}\left(\gamma_{-} r\right)$,

$\hat{w}=\frac{1}{2}\left[\tilde{G} I_{m+1}\left(\beta_{-} r\right)-G I_{m-1}\left(\beta_{-} r\right)\right]$

$$
-\frac{C m}{(k-\omega) r} I_{m}(k r)-\frac{A i m}{\operatorname{Re} \operatorname{Pr} r} I_{m}\left(\gamma_{-} r\right) \text {, }
$$

whereas for $r>1$ the solution with vanishing perturbations as $r \rightarrow \infty$ reduces to

$\hat{\rho}=B K_{m}\left(\gamma_{+} r\right)$,

$\hat{p}=D K_{m}(k r)+\frac{B \mathrm{i} S \omega}{\operatorname{Re}}\left(\mu_{V+}+\frac{4}{3} M-\frac{K}{\operatorname{Pr}}\right) K_{m}\left(\gamma_{+} r\right)$,

$\hat{u}=F K_{m}\left(\beta_{+} r\right)+\frac{D S k}{\omega} K_{m}(k r)-\frac{B \mathrm{i} K S^{2} k}{\operatorname{Re} \operatorname{Pr}} K_{m}\left(\gamma_{+} r\right)$,

$\hat{v}=\hat{w}+H K_{m-1}\left(\beta_{+} r\right)+\frac{D S k}{\omega} K_{m-1}(k r)$

$$
-\frac{B \mathrm{i} K S^{2} \gamma_{+}}{\operatorname{Re} \operatorname{Pr}} K_{m-1}\left(\gamma_{+} r\right)
$$

$\hat{w}=\frac{1}{2}\left[\tilde{H} K_{m+1}\left(\beta_{+} r\right)-H K_{m-1}\left(\beta_{+} r\right)\right]$

$$
+\frac{D S m}{\omega r} K_{m}(k r)-\frac{B \mathrm{i} K S^{2} m}{\operatorname{Re} \operatorname{Pr} r} K_{m}\left(\gamma_{+} r\right) \text {. }
$$

In the formulation, $I_{m}$ and $K_{m}$ represent the modified Bessel functions of order $m$ and

$$
\begin{aligned}
& \beta_{-}=\sqrt{k^{2}+\mathrm{iRe}(k-\omega)}, \quad \beta_{+}=\sqrt{k^{2}-\mathrm{i} \operatorname{Re} \omega /(S M)}, \\
& \gamma_{-}=\sqrt{k^{2}+\mathrm{i} \operatorname{Re} \operatorname{Pr}(k-\omega)}, \quad \gamma_{+}=\sqrt{k^{2}-\mathrm{i} \operatorname{Re} \operatorname{Pr} \omega /(S K)},
\end{aligned}
$$

are the values of $\beta$ and $\gamma$ in the regions of uniform base flow, the minus and plus signs in the subscripts indicating the regions $0 \leq r<1$ and $r>1$, respectively.

The solution depends on the ten integration constants $(A, B, C$, $D, E, F, G, \tilde{G}, H, \tilde{H})$. Evaluating the continuity equation (3a) twice, by using (14a)-(14e) for $0 \leq r<1$ and (15a)-(15e) for $r>1$ provides the pair of equations

$k E+\beta_{-}(\tilde{G}+G) / 2=0$,

$k F-\beta_{+}(\tilde{H}+H) / 2=0$.

Additional relationships, given below in (20a)-(20h), are obtained by matching the two sets of solutions at $r=1$ through appropriate jump conditions, to be derived from repeated integrals of the conservation equations (3a)-(3e) across the discontinuity. The resulting integrals are evaluated in the range $r=1 \pm \varepsilon$, with the jump conditions arising as the limiting forms of the resulting integrals when the limit $\varepsilon \rightarrow 0$ is taken for the semithickness $\epsilon$ of the integration domain. The methodology follows that employed by Drazin [5] in deriving jump conditions for the Orr-Sommerfeld equation, although the necessary analysis is somewhat more elaborate here because of the presence of a larger number of equations and variables, which in turn leads to a number of intricacies in the development.

As explained in Appendix B, the eight jump conditions to be satisfied by the disturbances across the discontinuity of the basic flow can be expressed in the form

$[\hat{u}]_{-}^{+}=-\bar{\kappa} \hat{T} \int_{-}^{+} \frac{U^{\prime} \bar{\mu}^{\prime}}{\bar{\mu} \bar{\kappa} \bar{T}^{\prime}} \mathrm{d} r$

$[\bar{\rho} \hat{v}]_{-}^{+}=0$

$[\hat{w}]_{-}^{+}=0$,

$[\bar{\kappa} \hat{T}]_{-}^{+}=0$

$\left[\bar{\mu} \hat{u}^{\prime}+U^{\prime} \hat{\mu}+k \bar{\mu} \hat{v}-\mathrm{iRe} U \bar{\rho} \hat{v}\right]_{-}^{+}=2 k \bar{\rho} \hat{v} \int_{-}^{+} \frac{\bar{\mu}^{\prime}}{\bar{\rho}} \mathrm{d} r$,

$\left[\frac{2 \bar{\mu}(r \hat{v})^{\prime}}{r}+\left(\bar{\mu}_{V}-\frac{2 \bar{\mu}}{3}\right)\left(k \hat{u}+\frac{(r \hat{v})^{\prime}}{r}+\frac{m \hat{w}}{r}\right)+\mathrm{iRe} \hat{p}\right]_{-}^{+}$

$=\frac{2 \bar{\rho} \hat{v}}{r} \int_{-}^{+} \frac{\bar{\mu}^{\prime}}{\bar{\rho}} \mathrm{d} r$

$\left[\bar{\mu}\left(\frac{\hat{w}}{r}\right)^{\prime}+m \bar{\mu} \hat{v}\right]_{-}^{+}=2 m \bar{\rho} \hat{v} \int_{-}^{+} \frac{\bar{\mu}^{\prime}}{\bar{\rho}} \mathrm{d} r$

$\left[\bar{\kappa} \hat{T}^{\prime}+\bar{T}^{\prime} \hat{\kappa}-\mathrm{iRe} \operatorname{Pr} \bar{T} \bar{\rho} \hat{v}\right]_{-}^{+}=0$.

In the notation, []$_{-}^{+}$is used to denote the jump of a given quantity across the discontinuity, so that for instance $[\hat{u}]_{-}^{+}=$ $\hat{u}(r=1+)-\hat{u}(r=1-)$. Correspondingly, $\int_{-}^{+}$indicates radial integration between $r=1-\varepsilon$ and $r=1+\varepsilon$ in the limit $\varepsilon \rightarrow 0$.

The boundary condition $(20 \mathrm{~h})$ states that the transverse transport rate of heat must be equal on both sides of the discontinuity, whereas (20e)-(20g) correspond to the equilibrium of normal and tangential stresses, and involve both the surface curvature and the variation of the base viscosity across the 
discontinuity. The conservation of mass flow rate across the discontinuity determines the jump of radial velocity $\hat{v}$ according to (20b), while the azimuthal perturbation velocity $\hat{w}$ does not change, as implied by (20c), and the streamwise disturbance $\hat{u}$ exhibits a jump when variations of base viscosity are considered, as observed in (20a). It is also of interest that changes in thermal conductivity lead to changes in temperature across the discontinuity, as seen in (20d). Clearly, while some of these results could have been anticipated from the beginning, others are less intuitive and require the careful derivation given in Appendix B.

As can be seen, the jump conditions involve different integrals of the base profiles across the discontinuity, which vanish only in flows with constant viscosity such that $\bar{\mu}^{\prime}=0$. A simple case arises when the density $\bar{\rho}$ is given as a function of $\bar{\mu}$ by specifying Eqs. (7)-(8) so that the integral $\int_{-}^{+}\left(\bar{\mu}^{\prime} / \bar{\rho}\right) \mathrm{d} r$ becomes a function of the boundary values of $\bar{\mu}$ at $r=1 \pm \varepsilon$, but is otherwise independent of the specific form of the profiles of density and viscosity across the discontinuity. The integral appearing on the right-hand side of (20a) is more complex in that it involves the derivative of the base velocity profile $U^{\prime}$, and therefore depends in principle on the shape of the base flow profiles across the discontinuity. Note that this unexpected dependence of the jump condition was also encountered by Drazin [15] in his stability analysis of incompressible parallel flow with variable density and viscosity.

Substituting (14a)-(15e) into the jump conditions (20a)-(20h) and using (18) and (19) to eliminate $E$ and $F$ provides a system of eight homogeneous linear equations for $(A, B, C, D, G, \tilde{G}, H, \tilde{H})$. Non-trivial solutions exist if the determinant of the associated coefficient matrix equals zero, thereby yielding the desired dispersion relation between $\omega$ and $k$ in terms of the parameters of the problem. The coefficient matrix can be simplified by eliminating terms through appropriate row and column linear combinations. The resulting dispersion relation is given in Appendix C. Note that the integrals in the jump conditions appear in the determinant under the form of $Q_{1}$ and $Q_{2}$, which have been simplified for the case where (7)-(8) are being used, anticipating the application of the discontinuous formalism in the next section.

\section{Application to the stability of non-isothermal round jets}

To illustrate the use of the formalism introduced in Section 3, let us consider its application to the study of the viscous stability properties of the unbounded non-isothermal axisymmetric jets formulated in Section 2, in the limit of vanishingly thin shear layers. As commented before, the parallel base flow is uniquely determined by the jet-to-ambient density ratio $S$ and a measure of the shear-layer thickness $\delta^{*}$, together with a pair of functions describing the profiles of base-flow velocity $U(r)$ and density $\bar{\rho}(r)$. The transverse profiles of the remaining physical properties are then given by the isobaric equation of state, $\bar{\rho}=1 / \bar{T}$, and the constitutive equations $\bar{\mu}(\bar{T}), \bar{\mu}_{V}(\bar{T})$ and $\bar{\kappa}(\bar{T})$. Notice that Eqs. (3a)-(3e) introduce ( $\operatorname{Re}, \operatorname{Pr}, \sigma, m)$ as additional parameters besides $S$ and $\theta$.

We also assume that the velocity and temperature profiles share common transverse length scales $a^{*}$ and $\delta^{*}$. In this case, it is now well known that both length scales are relevant in determining the linear spatiotemporal instability properties of the flow, the normal modes being naturally divided into shear layer and columnar modes [19,14,25,26]. The shear layer modes have cut-off wavenumbers $k^{*} \sim 1 / \delta^{*}$, characteristic frequencies $\omega^{*} \sim \delta^{*} / U_{0}^{*}$, and dominate the linear impulse response at group velocities $\mathrm{d} \omega^{*} / \mathrm{d} k^{*} \sim U_{0}^{*}$. In addition, their associated transverse eigenfunctions peak at the shear layer and decay rapidly away from it and are, thus, equivalent to the Kelvin-Helmholtz mode of a single shear layer. This fact was demonstrated by
Abid [27], where the shear layer modes of an inviscid compressible circular jet were studied by an asymptotic analysis in the limit $\delta^{*} / a^{*} \rightarrow 0$. In contrast with shear layer modes, columnar modes, of interest here, have characteristic wavenumbers $k^{*} \sim 1 / a^{*}$, characteristic frequencies $\omega^{*} \sim a^{*} / U_{0}^{*}$, and dominate the linear impulse response for group velocities $\mathrm{d} \omega^{*} / \mathrm{d} k^{*} \ll U_{0}^{*}$. Moreover, the amplitude of their associated eigenfunctions is finite at the centreline and, therefore, they do not have a counterpart in the case of a single shear layer.

As discussed by Drazin [5], the formalism developed in Section 3 is expected to describe the stability properties of base flows with continuous profiles only in the case of wavelengths much larger than the shear-layer thickness, $k^{*} \delta^{*} \rightarrow 0$. This means, in particular, that the use of the discontinuous approximation is restricted to the study of the low wavenumber region of the spectrum of shear layer modes, and is then useful to obtain approximations to the lower neutral curve, as Drazin [5] did for the particular cases of the two-dimensional mixing layer and jet. Here, however, we are mainly interested in the study of columnar modes, for which the discontinuous approach is particularly useful, since in this case $k=k^{*} a^{*} \sim 1 \Rightarrow k^{*} \delta^{*} \sim \delta^{*} / a^{*}=\delta$. Thus, for finite values of both $k$ and Re, the columnar eigenvalues obtained with continuous profiles are expected to converge uniformly to those associated with discontinuous profiles in the limit $\delta \rightarrow 0$.

To check the validity of the theory developed in Section 3 for discontinuous base profiles let us compare its predictions with calculations performed with the widely used parametric hyperbolic tangent profiles (12)-(13), introduced in Section 2. Note that, in this case, the use of (13) permits to evaluate the integral $Q_{1}$ appearing in the dispersion relation of Appendix $C$ to give

$Q_{1}=1-S^{-\sigma}$.

To solve the eigenvalue problem for continuous profiles, the stability equations (3a)-(3e) must be solved with the appropriate boundary conditions at $r=0$ and as $r \rightarrow \infty$ discussed in Section 2. To that end, we use a spectral collocation method similar to that employed by Lesshafft and Huerre [14]. In particular, the problem is written as $\boldsymbol{A} \boldsymbol{x}=\omega \boldsymbol{B} \boldsymbol{x}$ where $\boldsymbol{x}=(\hat{\rho}, \hat{u}, \hat{v}, \hat{w}, \hat{p})$, and $\boldsymbol{A}$ and $\boldsymbol{B}$ are two linear operators. The eigenfunctions $(\hat{\rho}, \hat{u}, \hat{v}, \hat{w}, \hat{p})$ are mapped from the physical domain $0 \leq r \leq r_{\max }$ onto the Chebyshev interval $-1 \leq \xi \leq 1$ using $\xi(r)=r_{c} /(2 r)-[1+$ $\left.r_{c}^{2} /\left(4 r^{2}\right)+2 r_{c} / r_{\max }-r_{c} / r\right]^{1 / 2}$, and then discretised in $N$ collocation points $\xi_{j}=\cos (j \pi /(N-1)), j=0 \ldots N-1$. By setting $r_{c}=2$ in the transformation, approximately half of the points $r_{j}=r\left(\xi_{j}\right)$ are placed in the interval $0 \leq r \leq 2$, concentrated around $r=1$. The number $N$ of collocation points is adapted so that a minimum of 10 collocation points are placed within the shear layer. The value of $r_{\text {max }}$ is increased with $N$, typical values lying in the range $120<N<1000,200<r_{\max }<1000$ for the results presented here. The linear operators $\boldsymbol{A}$ and $\boldsymbol{B}$ of the generalised eigenvalue problem are $5 N \times 5 N$-matrices containing $5(N-1)$ lines with the five stability equations discretised in the $N-1$ interior nodes, and $10 \mathrm{~N}$ lines representing the boundary conditions. The ZGGEV routine from the LAPACK library is used to compute $\omega$.

The solution of the dispersion relation of Appendix $C$, being an algebraic relation between $\omega$ and $k$, is carried out with a Newton-Raphson algorithm and is very time-efficient. Compared to the computation of an eigenvalue for the continuous stability problem for a small value of the momentum thickness, the computational time associated with the discontinuous formalism can be several orders of magnitude smaller.

\subsection{Temporal stability analysis}

To show how the limit of discontinuous base profiles is achieved as the shear-layer thickness decreases, let us begin 

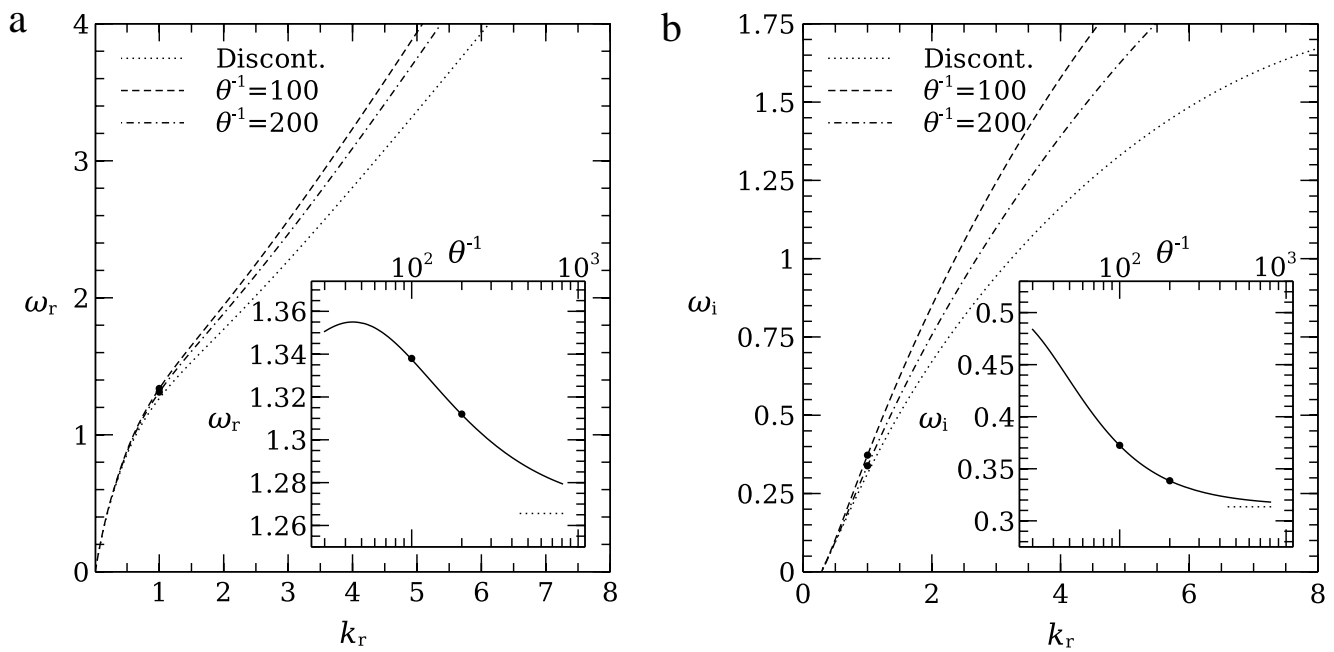

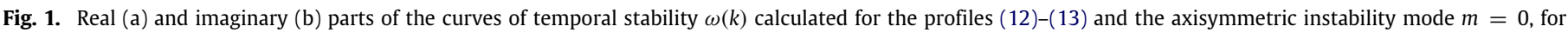

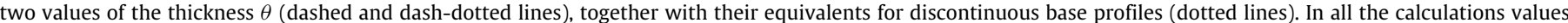

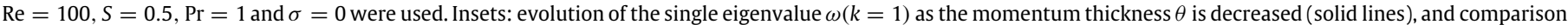
with its limiting value calculated with discontinuous base profiles (dotted line). The dots indicate corresponding points between the main plots and the insets.

by considering the unstable temporal stability branch of the axisymmetric instability mode $m=0$ for round jets with a density ratio $S=0.5$, in the case of constant viscosity and thermal conductivity, $\sigma=0$, as well as zero bulk viscosity $\bar{\mu}_{V}=0$. Fig. 1(a) and (b) display, respectively, the frequency, $\omega_{r}$, and growth rate, $\omega_{i}$, as functions of the real wavenumber, $k_{r}$, for values of $1 / \theta=$ $(100,200)$ (dashed and dash-dotted lines as indicated in the legend). Also shown as dotted curves are the corresponding results obtained by using the algebraic dispersion relation defined in Appendix C, obtained with the methodology described in Section 3 for discontinuous profiles. Notice that the instability modes shown in Fig. 1 are of the shear layer family, having group velocities of the order of the jet velocity, $\partial \omega_{r} / \partial k_{r} \sim \mathcal{O}(1)$.

The results shown in Fig. 1 are consistent with the behaviour expected from the discontinuous approximation. In particular, the low wavenumber end of the temporal spectrum obtained with continuous profiles, $\omega(k \rightarrow 0)$, is seen to converge to the discontinuous approximation, regardless of the value of $\theta$. Moreover, it is also observed that the upper limit of the range of wavenumbers which can be approximated with small relative errors by means of discontinuous profiles, increases as the shearlayer thickness decreases. For instance, notice in Fig. 1(b) that the value of $\omega_{i}$ obtained for the jet with $1 / \theta=100$ is approximated by the corresponding piecewise-constant base flow, with small relative errors that are less than $8 \%$ for values of the wavenumber up to $k \simeq 0.5$. This upper limit increases to $k \simeq 1$ in the case of a thinner profile with $1 / \theta=200$. To illustrate the convergence process more clearly, the insets displayed in Fig. 1 show the dependence on the shear-layer thickness of the single eigenvalue $\omega(k=1)$, together with its limiting value obtained by using discontinuous base profiles (dotted line), the solid circles indicating corresponding points in each subfigure. It is noteworthy that the temporal growth rates in Fig. 1(b) decrease as the shear layer becomes thinner. This behaviour can be explained by the fact that, although the Reynolds number Re is kept constant, the Reynolds number based on the shear-layer thickness, $\operatorname{Re} \theta$, decreases with $\theta$.

Finally, to illustrate the computational advantage of using the discontinuous formalism presented in this work, let us mention that the computation of a single eigenvalue for the jet with hyperbolic tangent base profiles and $\theta=1 / 200$ takes about $3 \mathrm{~min}$ on a $2.26 \mathrm{GHz}$ Intel Xeon processor, while it takes a few seconds using the dispersion relation corresponding to the discontinuous limit. For decreasing values of $\theta$ this difference becomes even more pronounced. In the next section, iterative procedures will be employed to study the transition from convective instability to absolute instability. Keeping in mind that to obtain a single point along the transition curve requires about 50-100 eigenvalue computations, the gain in computational time becomes evident.

\subsection{Spatiotemporal stability analysis and A/C transition}

A phenomenon of special interest in the study of parallel variable density jets is the transition from convective (C) instability to absolute (A) instability. It is now well established that the global dynamics observed in these flows is closely related with the absolute or convective character of the instability associated with their underlying base profiles; see for instance Refs. [28,29] and references therein. In the particular case of jets without coflow, there exists a critical value of the density ratio $S_{c}$ below which this transition takes place and which, in the case of the parametric base profiles given by Eqs. (12)-(13) is a function of the momentum thickness $\theta$.

In the limit of vanishingly thin shear layers, the use of the theory of discontinuous base profiles developed above allows the computation of the critical density ratio $S_{c}$ at which the A/C transition occurs. To show how the limit of discontinuous profiles is achieved, we calculated transition diagrams for the base profiles given by Eqs. (12)-(13), different instability modes, and Re = $\{100,200,2000\}$, decreasing the value of $\theta$ while carefully adjusting the number of collocation points to ensure a numerically converged solution. The $\mathrm{A} / \mathrm{C}$ transition curves were obtained by following the most unstable normal mode $\left(k^{(0)}, \omega^{(0)}\right)$ having zero group velocity, $\mathrm{d} \omega / \mathrm{d} k=0$, and accomplishing the Briggs-Bers pinching criterion; see for instance [30] for details. Negative values of the absolute growth rate, $\omega_{i}^{(0)}<0$, are associated with a convective instability, while positive ones, $\omega_{i}^{(0)}>0$, indicate an absolute instability. The transition curve $S_{c}(\theta)$ is then defined by the condition $\omega_{i}^{(0)}\left(S_{c}, \theta\right)=0$, and is tracked with a continuation method. At every step in $\theta$, the critical density ratio $S_{c}$ is found with a Newton-Raphson method, whereby the initial guess for $S_{c}$ and $\left(k^{(0)}, \omega^{(0)}\right)$ is obtained by extrapolating the last two obtained values.

The numerical method used to obtain the saddle point $\left(k^{(0)}, \omega^{(0)}\right)$ is that proposed by Deissler [31]. In the surroundings 


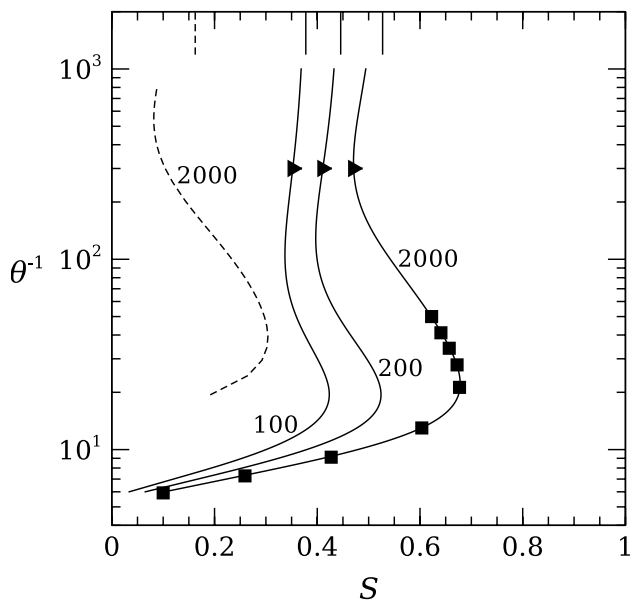

Fig. 2. Transition curves between convective and absolute instabilities in the $(S, 1 / \theta)$ plane for a hot round jet with base profiles (12)-(13), for the axisymmetric instability mode $m=0$ (solid lines) and the first helical mode $m=1$ (dashed line), with $\operatorname{Pr}=1, \sigma=0$, and different values of the Reynolds number Re = $(100,200,2000)$. The solid squares indicate the values calculated by Lesshafft and Huerre [14]. The corresponding values of the critical density ratio obtained with the dispersion relation of Appendix $\mathrm{C}$ for discontinuous base profiles are indicated by the straight lines in the upper part of the diagram. The triangles at $\theta=1 / 300$ correspond to those of Fig. 3.

of the saddle point $\left(k^{(0)}, \omega^{(0)}\right)$, the frequency $\omega(k)$ as a function of the wavenumber $k$ admits a quadratic Taylor expansion $\omega(k)=$ $\omega^{(0)}+l\left(k-k^{(0)}\right)^{2}$ around $k^{(0)}$. This can be exploited in an iterative procedure to find the location of $\left(k^{(0)}, \omega^{(0)}\right)$ as follows. Three wavenumbers $k_{1}, k_{2}, k_{3}$ are chosen around an initial guess $k_{*}^{(0)}$ with $k_{n}=k_{*}^{(0)}\left(1+\varepsilon \mathrm{e}^{\mathrm{i} 2 \pi(n-1) / 3}\right),(n=1,2,3)$, where $\varepsilon$ is a distance small enough for $k_{n}$ to fall in the region where the quadratic approximation of $\omega(k)$ is valid. Then the eigenvalue problem is solved for each of the $k_{n}$ using the spectral collocation method described before, yielding three frequencies $\omega_{1}, \omega_{2}$ and $\omega_{3}$. Now the three pairs $\left(k_{n}, \omega_{n}\right)$ can be used in a Newton-Raphson method to find $\left(k^{(0)}, \omega^{(0)}\right)$ and $l$ that accomplish $\omega_{n}-\omega^{(0)}-l\left(k_{n}-k^{(0)}\right)^{2}=0,(n=$ $1,2,3)$. With the newly obtained value of $k^{(0)}$, three new values of $k_{1}, k_{2}$ and $k_{3}$ are calculated, and the procedure is repeated until relative differences in $\left(k^{(0)}, \omega^{(0)}\right)$ between subsequent iteration steps fall below $10^{-5}$. At each step $\varepsilon$ is slightly decreased to enhance the accuracy of the procedure.

The results of these calculations are shown in Fig. 2, where the critical density ratio $S_{c}$ is given as a function of the inverse thickness parameter $1 / \theta$, for the axisymmetric base profiles specified by (12)-(13). Both the axisymmetric mode $m=0$ (solid lines) and the first helical mode $m=1$ (dashed line) are considered. The values of the critical density ratio obtained by using discontinuous base profiles are represented by the straight lines in the upper part of the diagram. All calculations were performed using $\operatorname{Pr}=1, \sigma=0$, and various values of the Reynolds number, $\operatorname{Re}=\{100,200,2000\}$, the latter selected to enable comparisons with the results obtained by Lesshafft and Huerre [14], indicated by the squares in Fig. 2. The perfect agreement provides further confidence on the accuracy of the numerical code used in the present work for continuous profiles.

Note that the transition curve shown in Fig. 2 for the axisymmetric mode and $\operatorname{Re}=2000$ (solid line) indicates, in particular, that the minimum value of $\theta=1 / 50$ employed by Lesshafft and Huerre [14] is not small enough to reproduce the limit of a vanishingly thin shear layer (straight line). Thus, in order to study how this limit is approached, in the present work we have extended the computations with continuous base profiles to values of the momentum thickness as small as $\theta \simeq 10^{-3}$. The results reveal that for decreasing values of $\theta$, the convergence of the function $S_{c}(\operatorname{Re})$ towards its vortex-sheet limit, i.e. the straight lines in the upper part

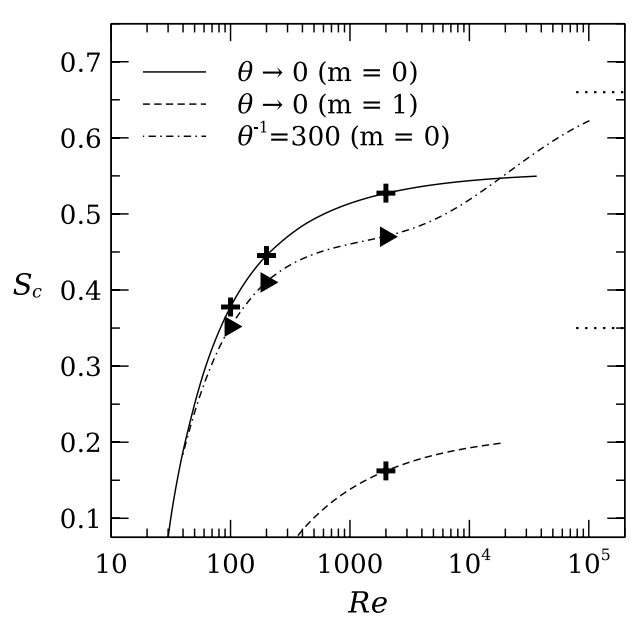

Fig. 3. The function $S_{c}(\mathrm{Re})$ given by the discontinuous approximation for $m=0$ (solid line) and $m=1$ (dashed line), and by computations for the continuous jet profiles (12)-(13) with $\theta=1 / 300$ for $m=0$ (dashed-dotted line). The crosses correspond to the limits indicated with straight lines in Fig. 2, while the locations of the triangles in the $(S, \theta)$-plane of Fig. 2 are indicated with the same symbols. The straight dotted lines on the right hand side of the diagram indicate the values $S_{c} \simeq 0.66$ and $S_{c} \simeq 0.35$ for $m=0$ and $m=1$, respectively, that correspond to an axisymmetric inviscid vortex sheet [11].

of the figure, is faster for smaller values of Re. This finding seems to be in agreement with the approximations made in deriving the jump conditions (20a)-(20h), which neglect the contributions of various integrals across the shear layer that are proportional to Re, appearing on the right-hand sides of (B.1a)-(B.3c). Consequently, for a given shear-layer thickness, the errors involved in the vortexsheet approximation increase with the Reynolds number and lead to the slower convergence shown in Fig. 2. It is also worth remarking the non-monotonic behaviour of the transition curves as the shear-layer thickness decreases to values of $\theta<1 / 50$, a range unexplored in the literature, which includes a pronounced decrease in $S_{c}$, followed by a minimum and an inflection point, before reaching the corresponding vortex-sheet limit, $\theta \rightarrow 0$. This finite Reynolds number behaviour is in marked contrast with its inviscid counterpart, whose corresponding curve $S_{c}(\theta)$ decreases monotonically towards the vortex-sheet limiting value $S_{c}(0)$ as $\theta$ decreases, giving negligible departures $S_{c}(\theta)-S_{c}(0)$ for values of the shear-layer thickness $\theta \lesssim 10^{-2}$ (see for instance $[19,14,32]$ ).

The virtue of the thin-shear layer approximation becomes clear when studying parametric variations of the stability properties of the jet flow. As an example, in Fig. 3 we show the critical density ratio $S_{c}$ as a function of the Reynolds number Re, in the limit of vanishingly thin shear layers. Both the axisymmetric mode $m=0$ (solid line) and the first helical mode $m=1$ (dashed line) were obtained with the dispersion relation of Appendix C, in a very timeefficient way. The crosses along these lines correspond to the limits indicated with straight lines in Fig. 2. For $m=0$, the curve $S_{c}(\mathrm{Re})$ is compared to its counterpart from a stability calculation using the continuous hyperbolic profiles (12)-(13) with momentum thickness $\theta=1 / 300$ (dashed-dotted line). The triangles along this curve correspond to the triangles in the $(S, \theta)$-plane of Fig. 2 . Moving vertically in Fig. 3 from a triangle to a cross thus corresponds to moving upwards along the respective transition curve in Fig. 2 from the triangle to the limit $\theta \rightarrow 0$. The distance in Fig. 3 between triangles and crosses increases for increasing Reynolds numbers. This is in agreement with the fact that in Fig. 2 the limit of infinitesimally thin shear layers is achieved at a slower rate for higher Reynolds numbers.

When the Reynolds number is further increased, the dashed-dotted curve $S_{c}(\operatorname{Re})$ for $m=0$ and $\theta=1 / 300$ is seen to converge to its inviscid limit, which is expected to lie in the range 
$0.66 \lesssim S_{c} \lesssim 0.70$ (see Fig. 6 of Ref. [14] and Fig. 4 of Ref. [32]). Note that the lower limit of this range, $S_{c} \simeq 0.66$, is the critical density ratio for an axisymmetric inviscid vortex sheet, and is indicated, together with its counterpart for $m=1, S_{c} \simeq 0.35$, by a straight dotted line on the right hand side of Fig. 3 . It is also interesting to note the non-monotonic convergence of the dashed-dotted line to its inviscid limit when $\mathrm{Re} \rightarrow \infty$, showing a rather complicated behaviour featuring a pair of inflection points at $\mathrm{Re} \simeq 1000$ and at $\operatorname{Re} \simeq 2 \times 10^{4}$.

Finally, let us point out that the results shown in Fig. 3 are in agreement with Drazin [5] who pointed out that the viscous dispersion relation obtained for vanishingly thin shear layers should not be expected to reproduce, in the limit $\operatorname{Re} \rightarrow \infty$, the results given by the inviscid vortex sheet. Indeed, the solid curve $S_{c}(\mathrm{Re})$ shown in Fig. 3 does not converge to the inviscid critical density ratio $S_{c} \simeq 0.66$ for $m=0$, as neither does the dashed curve for $m=1$. To explain this apparent contradiction, we emphasise that in the derivation of the jump conditions, presented in Appendix B, the value of $k /$ Re is kept finite. This yields jump conditions that guarantee, inter alia, equilibrium of tangential stresses at the discontinuity. However, if in our derivation we let $\operatorname{Re} \rightarrow \infty$ from the start, we would obtain instead the jump conditions corresponding to an inviscid vortex sheet, which express, besides continuity of pressure, that the boundary is a material surface. The different physical nature of the viscous and inviscid jump conditions gives an indication of why the inviscid vortex sheet is a singular limit that cannot be recovered, within the present formulation, by letting $\operatorname{Re} \rightarrow \infty$ a posteriori.

\section{Conclusions}

We have presented a simple methodology appropriate to study the viscous linear stability properties of variable-density parallel jet flows with very thin shear layers, including the effect of variable transport properties, thereby providing a natural generalisation of the formalism originally developed by Drazin $[5,15]$ to the case of low-Mach-number gaseous jets. This discontinuous representation of the base flow, as discussed by Drazin [5], is useful to study modal solutions with wavelengths much larger than the characteristic shear-layer thickness. The methodology includes the use of successive integrations of the stability equations across the vortex sheet to derive the jump conditions that serve to match the solutions of the stability problem on both sides of the discontinuity. In particular, we have applied the formalism to axisymmetric jets that, in the limit of very thin shear layers, can be represented by piecewise constant base profiles. Analytic solutions are found for the flow perturbations in the regions of constant velocity and density. Imposing the jump conditions across the discontinuity leads to a dispersion relation in the form of a closed algebraic expression that involves all the relevant parameters of the problem, which can easily be solved with a numerical rootfinding method to study the linear stability properties of the flow.

To validate the dispersion relation, we have compared its solutions with stability calculations performed for continuous thin-shear-layer base profiles. In particular, we have used in the comparisons the parametric hyperbolic tangent base profiles, commonly used in the literature to model axisymmetric jets. Our results reveal that the limit of discontinuous base profiles is successfully recovered as the thickness of the continuous base profiles decreases, both for temporal and spatiotemporal stability analyses. In addition, the discontinuous formalism is shown to be particularly useful when performing parametric studies for jets with very thin shear layers, in that the required computational times are several orders of magnitude smaller than those associated with continuous profiles.

The formalism was used to analyse gaseous jets with constant molecular weight, for which the density and transport properties are functions of the temperature. However, the formulation could be readily extended to account for molecular-weight variations by describing also the composition field, as discussed in the text above Eq. (9). In the case of an isothermal binary mixture of fluids, a situation frequently encountered in experiments and applications, the formulation is very simple, in that with uniform temperature the density variations can be computed in terms of the mass fraction of one of the two species, yielding for instance $\bar{\rho}=1 /[\bar{Y}+S(1-\bar{Y})]$, with $\bar{Y}$ denoting the base-flow mass fraction of the gas located near the axis and $S=\rho_{0}^{*} / \rho_{\infty}^{*}$ being the molecular-weight ratio for the binary mixture. The analysis necessitates the introduction of minor modifications to the coefficient matrix displayed in Appendix $\mathrm{C}$, as required to accommodate changes in the jump conditions (20d) and (20h), which should be replaced with $[\bar{\rho} \hat{Y}]_{-}^{+}=0$ and $\left[\bar{\rho} \hat{Y}^{\prime}+\bar{Y}^{\prime} \hat{\rho}-\operatorname{iRe}_{c} \bar{Y} \bar{\rho} \hat{v}\right]_{-}^{+}=0$, respectively, and also from changes in the eigenfunctions defined in Eqs. (15b)-(15e) and (17), where the thermal conductivity ratio $K$ should be replaced with $S^{-1}$. The resulting modified dispersion relation could be employed, for instance, to study the viscous stability characteristics of helium jets discharging into air, a configuration often used in experiments as a prototypical model for light jets.

The development indicates that for gaseous jets with variable viscosity the vortex-sheet limit exhibits a non-negligible dependence on the specific shape of the shear-layer profiles of velocity and density through the integrals appearing on the right-handsides of (20a) and (20e)-(20g), giving a result similar to that found by Drazin [15]. This finding suggests that, even in configurations with thin shear layers, care should be exerted when using model base profiles - as those given above in (12) and (13) - for the quantitative assessment of the viscous instability of gaseous jets and wakes. For instance, if accurate results are desired in the stability analysis of hot gas jets emerging from short injectors, investigated previously in the inviscid limit [32], then the computation should account for the Blasius velocity profile found near the injector wall, which would enter in the vortex-sheet limit through the integral contribution in (20a).

\section{Acknowledgements}

This work was supported by Spanish MCINN through the project CONSOLIDER \#CSD2010-00010, and the projects \#DPI201128356-C03-02 and \#ENE2008-06515-C04-01, and by the Comunidad de Madrid through projects \#S2009/ENE-1597 and \#CCG10-UC3M/DPI-4777.

\section{Appendix A. Stability eigenproblem with piecewise-constant base profiles}

In the regions of constant base profiles, the linearised stability equations (2a)-(2c) can be simplified to

$$
\begin{aligned}
& \frac{\partial \rho}{\partial t}+U \frac{\partial \rho}{\partial x}=-\bar{\rho} \nabla \cdot \boldsymbol{u}, \\
& \bar{\rho}\left(\frac{\partial \boldsymbol{u}}{\partial t}+U \frac{\partial \boldsymbol{u}}{\partial x}\right) \\
& =-\nabla p+\frac{1}{\operatorname{Re}}\left[\bar{\mu} \nabla^{2} \boldsymbol{u}+\left(\bar{\mu}_{V}+\frac{\bar{\mu}}{3}\right) \nabla(\nabla \cdot \boldsymbol{u})\right], \\
& \bar{\rho}\left(\frac{\partial \rho}{\partial t}+U \frac{\partial \rho}{\partial x}\right)=\frac{1}{\operatorname{Re} \operatorname{Pr}} \bar{\kappa} \nabla^{2} \rho .
\end{aligned}
$$

The energy equation (A.1c), which has been written for the density by using a generic equation of state $T(\rho)$, becomes, in normal modes,

$\hat{\nabla}_{\gamma, m}^{2} \hat{\rho}=0$ 
in terms of the Laplacian operator defined in (4), with $\gamma$ given in (6). An equation for $\hat{p}$ can be obtained by taking the divergence of the momentum equation (A.1b) and using the continuity equation (A.1a) together with (A.2) to give

$$
\hat{\nabla}_{k, m}^{2} \hat{p}=(k U-\omega)^{2}\left[\bar{\kappa}^{-1}\left(\bar{\mu}_{V}+4 \bar{\mu} / 3\right) \operatorname{Pr}-1\right] \hat{\rho} .
$$

On the other hand, substituting $\nabla \cdot \boldsymbol{u}$ from the continuity equation (A.1a) in the momentum equation (A.1b) yields

$$
\begin{aligned}
& \hat{\nabla}_{\beta, m}^{2} \hat{u}=\frac{i \operatorname{Re} k}{\bar{\mu}} \hat{p}-\frac{k(k U-\omega)}{\bar{\rho}}\left(\frac{1}{3}+\frac{\bar{\mu}_{V}}{\bar{\mu}}\right) \hat{\rho}, \\
& \hat{\nabla}_{\beta, m}^{2} \hat{v}-\frac{1}{r^{2}}(\hat{v}+2 m \hat{w}) \\
& =-\frac{i \operatorname{Re}}{\bar{\mu}} \hat{p}^{\prime}+\frac{k U-\omega}{\bar{\rho}}\left(\frac{1}{3}+\frac{\bar{\mu}_{V}}{\bar{\mu}}\right) \hat{\rho}^{\prime}, \\
& \hat{\nabla}_{\beta, m}^{2} \hat{w}-\frac{1}{r^{2}}(\hat{w}+2 m \hat{v}) \\
& =\frac{i \operatorname{Re} m}{r \bar{\mu}} \hat{p}-\frac{m(k U-\omega)}{r \bar{\rho}}\left(\frac{1}{3}+\frac{\bar{\mu}_{V}}{\bar{\mu}}\right) \hat{\rho}
\end{aligned}
$$

where $\beta$ is defined in (5). It is useful to add and subtract the latter two Eqs. (A.4b) and (A.4c), yielding

$$
\begin{aligned}
& \hat{\nabla}_{\beta, m+1}^{2}(\hat{v}+\hat{w}) \\
& \quad=-\frac{i \operatorname{Re}}{\bar{\mu}}\left(\hat{p}^{\prime}-\frac{m}{r} \hat{p}\right)+\frac{k U-\omega}{\bar{\rho}}\left(\frac{1}{3}+\frac{\bar{\mu}_{V}}{\bar{\mu}}\right)\left(\hat{\rho}^{\prime}-\frac{m}{r} \hat{\rho}\right), \\
& \hat{\nabla}_{\beta, m-1}^{2}(\hat{v}-\hat{w}) \\
& =-\frac{i \operatorname{Re}}{\bar{\mu}}\left(\hat{p}^{\prime}+\frac{m}{r} \hat{p}\right)+\frac{k U-\omega}{\bar{\rho}}\left(\frac{1}{3}+\frac{\bar{\mu}_{V}}{\bar{\mu}}\right)\left(\hat{\rho}^{\prime}+\frac{m}{r} \hat{\rho}\right) .
\end{aligned}
$$

Eqs. (A.2)-(A.4a) and (A.5a), (A.5b), together with appropriate boundary conditions enable the eigenfunctions of the disturbances to be determined sequentially. In particular, Eq. (A.2) can be solved for $\hat{\rho}$ to give the profiles shown in (14a) and (15a). The result can be substituted into (A.3), thereby enabling the pressure profiles (14b) and (15b) to be computed. Finally, substitution of $\hat{\rho}$ and $\hat{p}$ into (A.4a), (A.5a) and (A.5b) provides the profiles defined in $(14 c)-(14 e)$ and $(15 c)-(15 e)$ upon integration.

\section{Appendix B. Boundary conditions for the disturbances at a discontinuity of the base flow}

The derivation of the jump conditions at $r=1$ begins by integrating once the stability equations (3a)-(3e) after multiplication by $r$ to yield

$$
\begin{aligned}
& r \bar{\rho} \hat{v}=-\int r(k U-\omega) \hat{\rho} \mathrm{d} r-k \int r \bar{\rho} \hat{u} \mathrm{~d} r-m \int \bar{\rho} \hat{w} \mathrm{~d} r, \\
& r\left(\bar{\mu} \hat{u}^{\prime}+U^{\prime} \hat{\mu}+k \bar{\mu} \hat{v}-\mathrm{i} \operatorname{Re} U \bar{\rho} \hat{v}-2 k \bar{\rho} \hat{v} \int \frac{\bar{\mu}^{\prime}}{\bar{\rho}} \mathrm{d} r\right) \\
& =\int r\left(\beta^{2}+\frac{m^{2}}{r^{2}}\right) \bar{\mu} \hat{u} \mathrm{~d} r \\
& \quad+k \int r\left[\frac{2 \bar{\mu}(r \hat{v})^{\prime}}{r}+\left(\bar{\mu}_{V}-\frac{2 \bar{\mu}}{3}\right) \frac{(r \hat{v})^{\prime}}{r}+\mathrm{iRe} \hat{p}\right] \mathrm{d} r \\
& -\mathrm{iRe} \int U(r \bar{\rho} \hat{v})^{\prime} \mathrm{d} r+k \int\left(\bar{\mu}_{V}+\frac{\bar{\mu}}{3}\right)(r k \hat{u}+m \hat{w}) \mathrm{d} r \\
& \quad-2 k \int(r \bar{\rho} \hat{v})^{\prime} \int \frac{\bar{\mu}^{\prime}}{\bar{\rho}} \mathrm{d} r \mathrm{~d} r, \\
& r\left[\frac{2 \bar{\mu}(r \hat{v})^{\prime}}{r}+\left(\bar{\mu}_{V}-\frac{2 \bar{\mu}}{3}\right)\left(k \hat{u}+\frac{(r \hat{v})^{\prime}}{r}+\frac{m \hat{w}}{r}\right)+\mathrm{iRe} \hat{p}\right]
\end{aligned}
$$

$$
\begin{aligned}
& -2 \bar{\rho} \hat{v} \int \frac{\bar{\mu}^{\prime}}{\bar{\rho}} \mathrm{d} r \\
& =\int r\left(\beta^{2}+\frac{m^{2}}{r^{2}}\right) \bar{\mu} \hat{v} \mathrm{~d} r-\int r k\left(\bar{\mu} \hat{u}^{\prime}+U^{\prime} \hat{\mu}\right) \mathrm{d} r \\
& +\int\left[\frac{2 \bar{\mu}(r \hat{v})^{\prime}}{r}+\left(\bar{\mu}_{V}-\frac{2 \bar{\mu}}{3}\right)\left(k \hat{u}+\frac{(r \hat{v})^{\prime}}{r}+\frac{m \hat{w}}{r}\right)\right. \\
& +\mathrm{iRe} \hat{p}] \mathrm{d} r+3 m \int \frac{\bar{\mu} \hat{w}}{r} \mathrm{~d} r-m \int \bar{\mu} \hat{w}^{\prime} \mathrm{d} r \\
& -2 \int(\bar{\rho} \hat{v})^{\prime} \int \frac{\bar{\mu}^{\prime}}{\bar{\rho}} \mathrm{d} r \mathrm{~d} r, \\
& r^{2} \bar{\mu}\left(\frac{\hat{w}}{r}\right)^{\prime}+m \bar{\mu} \hat{v}-2 m \bar{\rho} \hat{v} \int \frac{\bar{\mu}^{\prime}}{\bar{\rho}} \mathrm{d} r \\
& =\int r\left(\beta^{2}+\frac{m^{2}}{r^{2}}\right) \bar{\mu} \hat{w} \mathrm{~d} r+\int \frac{1}{r}\left[m \bar{\mu} \hat{v}-r^{2} \bar{\mu}\left(\frac{\hat{w}}{r}\right)^{\prime}\right] \mathrm{d} r \\
& +m \int\left(\bar{\mu}_{V}+\frac{\bar{\mu}}{3}\right)\left(k \hat{u}+\frac{m \hat{w}}{r}\right) \mathrm{d} r \\
& +m \int\left[\frac{2 \bar{\mu}(r \hat{v})^{\prime}}{r}+\left(\bar{\mu}_{V}-\frac{2 \bar{\mu}}{3}\right) \frac{(r \hat{v})^{\prime}}{r}+\mathrm{iRe} \hat{p}\right] \mathrm{d} r \\
& -2 m \int(\bar{\rho} \hat{v})^{\prime} \int \frac{\bar{\mu}^{\prime}}{\bar{\rho}} \mathrm{d} r \mathrm{~d} r, \\
& r\left(\bar{\kappa} \hat{T}^{\prime}+\bar{T}^{\prime} \hat{\kappa}-\mathrm{iRe} \operatorname{Pr} \bar{T} \bar{\rho} \hat{v}\right) \\
& =\int r\left(\gamma^{2}+\frac{m^{2}}{r^{2}}\right) \bar{\kappa} \hat{T} \mathrm{~d} r-\mathrm{i} \operatorname{Re} \operatorname{Pr} \int \bar{T}(r \bar{\rho} \hat{v})^{\prime} \mathrm{d} r .
\end{aligned}
$$

Integration by parts has been used to arrange the equations so that all of the integrands appearing in the right-hand-side integrals remain finite within the discontinuity, and therefore give a vanishing contribution when the integrals are evaluated in the range $r=1 \pm \varepsilon$ and the limit $\varepsilon \rightarrow 0$ is considered. As a consequence, the resulting jump conditions emerging from these first quadratures involve only the terms on the left-hand side. This can be clearly seen, for instance, in the first quadrature of the continuity equation, given in (B.1a), where the base flow profiles and the disturbances $\hat{\rho}, \hat{u}$ and $\hat{w}$ appearing in the right-hand-side integrals can be anticipated to be finite, thereby resulting in a vanishing contribution in the limit $\varepsilon \rightarrow 0$. The continuity balance (B.1a) therefore requires that the transverse mass flux $\bar{\rho} \hat{v}$ takes equal values on both sides of the discontinuity, a condition that will be indicated by $[\bar{\rho} \hat{v}]_{-}^{+}=0$.

This condition of continuity of $\bar{\rho} \hat{v}$ can be used to prove that the last integral in (B.1e) also gives a vanishing contribution in the limit $\varepsilon \rightarrow 0$, so that the jump condition $\left[\bar{\kappa} \hat{T}^{\prime}+\bar{T}^{\prime} \hat{\kappa}-\operatorname{iRePr} \bar{T} \bar{\rho} \hat{v}\right]_{-}^{+}=$ 0 follows, because the second last integral also vanishes in the limit $\varepsilon \rightarrow 0$ provided $\hat{T}$ remains finite. Since in the present approximation the thermal conductivity is only a function of the temperature, its disturbances and those of the temperature are related by the equation $\hat{\kappa} / \bar{\kappa}^{\prime}=\hat{T} / \bar{T}^{\prime}$, which can be used in a second quadrature of (B.1e) to give

$$
\begin{aligned}
\bar{\kappa} \hat{T}= & \operatorname{iRe} \operatorname{Pr} \int \bar{T} \bar{\rho} \hat{v} \mathrm{~d} r+\int \frac{1}{r} \int r\left(\gamma^{2}+\frac{m^{2}}{r^{2}}\right) \bar{\kappa} \hat{T} \mathrm{~d} r \\
& -i \operatorname{Re} \operatorname{Pr} \int \frac{1}{r} \int \bar{T}(r \bar{\rho} \hat{v})^{\prime} \mathrm{d} r \mathrm{~d} r
\end{aligned}
$$

thereby leading naturally to the additional jump condition $[\bar{\kappa} \hat{T}]_{-}^{+}=0$ when the limit $\varepsilon \rightarrow 0$ is taken. 


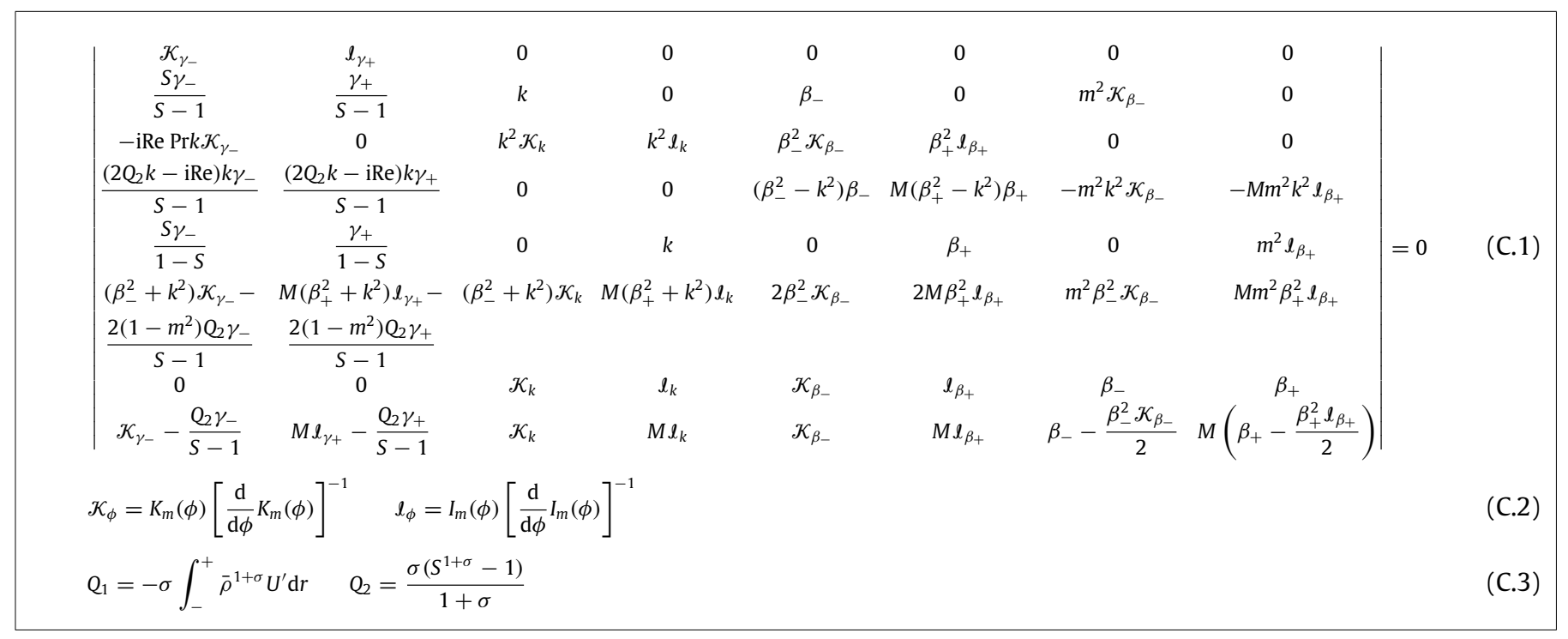

Box I.

For those terms on the right-hand sides of (B.1b)-(B.1d) that involve functions that might be expected to be singular, such as $\hat{v}^{\prime}$, or derivatives of discontinuous functions, such as $U^{\prime}$, proving the vanishing of the associated integrals necessitates additional information arising from second quadratures. Dividing (B.1b)-(B.1d) by $r \bar{\mu}, r$ and $r^{2} \bar{\mu}$, respectively, and integrating yields

$$
\begin{aligned}
\hat{u}+ & \int \frac{U^{\prime} \hat{\mu}}{\bar{\mu}} \mathrm{d} r \\
= & -k \int \hat{v} \mathrm{~d} r+\mathrm{i} \operatorname{Re} \int \frac{U \bar{\rho} \hat{v}}{\bar{\mu}} \mathrm{d} r+2 k \int \frac{\bar{\rho} \hat{v}}{\bar{\mu}} \int \frac{\bar{\mu}^{\prime}}{\bar{\rho}} \mathrm{d} r \mathrm{~d} r \\
& +\int \frac{1}{r \bar{\mu}}[\text { terms on r.h.s. of equation (B.1b)] } \mathrm{d} r \\
\int[ & \left.\frac{2 \bar{\mu}(r \hat{v})^{\prime}}{r}+\left(\bar{\mu}_{V}-\frac{2 \bar{\mu}}{3}\right)\left(k \hat{u}+\frac{(r \hat{v})^{\prime}}{r}+\frac{m \hat{w}}{r}\right)+\mathrm{iRe} \hat{p}\right] \mathrm{d} r \\
= & \int \frac{2 j \bar{\rho} \hat{v}}{r} \int \frac{\bar{\mu}^{\prime}}{\bar{\rho}} \mathrm{d} r \mathrm{~d} r \\
& +\int \frac{1}{r}[\text { terms on r.h.s. of equation (B.1c)] } \mathrm{d} r \\
\hat{w}= & -m r \int \frac{\hat{v}}{r^{2}} \mathrm{~d} r+2 m r \int \frac{\bar{\rho} \hat{v}}{r^{2} \bar{\mu}} \int \frac{\bar{\mu}^{\prime}}{\bar{\rho}} \mathrm{d} r \mathrm{~d} r \\
& +r \int \frac{1}{r^{2} \bar{\mu}}[\text { terms on r.h.s. of equation (B.1d)] } \mathrm{d} r .
\end{aligned}
$$

Again, it can be seen that all of the single integrals that appear on the right-hand side vanish in the limiting procedure used here, as their integrands at most experience finite jumps across the discontinuity layer. Furthermore, all of the terms that involve double integrals of the form $\int f(r) \int g(r) \mathrm{d} r \mathrm{~d} r$ can also be shown to vanish since both $f(r)$ and $\int g(r) \mathrm{d} r$ at most experience finite jumps at $r=1$. Consequently, in the limit $\varepsilon \rightarrow 0$, (B.3a) and (B.3c) provide the jump conditions $[\hat{u}]_{-}^{+}=-\int_{1-\varepsilon}^{1+\varepsilon}\left(U^{\prime} \hat{\mu} / \bar{\mu}\right) \mathrm{d} r$ and $[\hat{w}]_{-}^{+}=0$, respectively, while (B.3b) indicates that the integral on its left-hand side vanishes when evaluated between $1-\varepsilon$ and $1+\varepsilon$. Bearing this new information in mind it is straightforward to prove now that all of the integrals appearing on the right-hand side of (B.1b)-(B.1d) indeed vanish in the limit $\varepsilon \rightarrow 0$, so that their associated jump conditions involve only the terms on the lefthand side, as previously anticipated. This therefore completes the derivation of the set of eight jump conditions to be satisfied by the disturbances across the discontinuity of the basic flow, which are given in the main text in (20a)-(20h).

Note that integration by parts is employed in writing the integral $-\int_{1-\varepsilon}^{1+\varepsilon}\left(U^{\prime} \hat{\mu} / \bar{\mu}\right) \mathrm{d} r$ appearing on the right-hand side of (20a). For the jet of constant molecular weight, the equation $\hat{\mu}=\bar{\mu}^{\prime} \hat{T} / \bar{T}^{\prime}$, corresponding to the viscosity being expressible as a function of the temperature, must be used for the integration, along with the condition that the heat flux derivative $(\bar{\kappa} \hat{T})^{\prime}$ be finite across the discontinuity, as implied by the first equation in (20d), which is also used in evaluating the resulting integral.

\section{Appendix C. Determinant}

See Box I.

\section{References}

[1] H. Helmholtz, Über discontinuirliche Flüssigkeitsbewegungen, Monats. Königl. Preuss. Akad. Wiss. Berlin 23 (1868) 215-228.

[2] L. Kelvin, Hydrokinetic solutions and observations, Phil. Mag. Ser. 442 (1871) 362-377.

[3] L. Rayleigh, On the stability, or instability, of certain fluid motions, Proc. Lond. Math. Soc. (3) 11 (1880) 57-70.

[4] R.E. Esch, The instability of a shear layer between two parallel streams, J. Fluid Mech. 3 (1957) 289-303.

[5] P.G. Drazin, Discontinuous velocity profiles for the Orr-Sommerfeld equation, J. Fluid Mech. 10 (1961) 571-583.

[6] T. Tatsumi, K. Gotoh, Stability of free boundary layers between two streams, J. Fluid Mech. 7 (1960) 433-441.

[7] T. Tatsumi, T. Kakutani, The stability of a two-dimensional laminar jet, J. Fluid Mech. 4 (1958) 261-275.

[8] P. Huerre, P.A. Monkewitz, Local and global instabilities in spatially developing flows, Annu. Rev. Fluid Mech. 22 (1990) 473-537.

[9] P. Huerre, P.A. Monkewitz, Absolute and convective instabilities in free shear layers, J. Fluid Mech. 159 (1985) 151-168.

[10] P.A. Monkewitz, The absolute and convective nature of instability in twodimensional wakes at low Reynolds numbers, Phys. Fluids 31 (1988) 999-1006.

[11] P.A. Monkewitz, K.D. Sohn, Absolute instability in hot jets, AIAA J. 28 (1988) 911-916.

[12] M.-H. Yu, P.A. Monkewitz, The effect of nonuniform density on the absolute instability of two-dimensional inertial wakes and jets, Phys. Fluids A 2 (1990) 611-639.

[13] D.W. Lim, L.G. Redekopp, Absolute instability conditions for variable density, swirling jet flows, Eur. J. Mech. B Fluids 17 (1998) 165-185.

[14] L. Lesshafft, P. Huerre, Linear impulse response in hot round jets, Phys. Fluids 19 (2007) 024102.

[15] P.G. Drazin, On stability of parallel flow of an incompressible fluid of variable density and viscosity, Proc. Cambridge Philos. Soc. 58 (1962) 646-661.

[16] F.A. Williams, Combustion Theory, second ed., Benjamin Cummings, Menlo Park, CA, 1985

[17] P.A. McMurtry, W.H. Jou, J.J. Riley, R.W. Metcalfe, Direct numerical simulations of a reacting mixing layer with chemical heat release, AIAA J. 24 (1986) 962-970. 
[18] G.K. Batchelor, A.E. Gill, Analysis of the stability of axisymmetric jets, J. Fluid Mech. 14 (1962) 529-551.

[19] S. Jendoubi, P.J. Strykowski, Absolute and convective instability of axisymmetric jets with external flow, Phys. Fluids 6 (1994) 3000-3009.

[20] A. Sevilla, J.M. Gordillo, C. Martínez-Bazán, The effect of the diameter ratio on the absolute and convective instability of free coflowing jets, Phys. Fluids 14 (2002) 3028-3038

[21] M.P. Hallberg, V. Srinivasan, P. Gorse, P.J. Strykowski, Suppression of global modes in low-density axisymmetric jets using coflow, Phys. Fluids 19 (2007) 014102.

[22] J.W. Nichols, P.J. Schmid, J.J. Riley, Self-sustained oscillations in variabledensity round jets, J. Fluid Mech. 582 (2007) 341-376.

[23] K. Srinivasan, M.P. Hallberg, P.J. Strykowski, Viscous linear stability of axisymmetric low-density jets: parameters influencing absolute instability, Phys. Fluids 22 (2010) 024103.

[24] M.R. Khorrami, M.R. Malik, R.L. Ash, Applications of spectral collocation techniques to the stability of swirling flows, J. Comput. Phys. 81 (1989) 206.
[25] M.P. Juniper, The full impulse response of two-dimensional jet/wake flows and implications for confinement, J. Fluid Mech. 590 (2007) 163-185.

[26] P. Meliga, D. Sipp, J.-M. Chomaz, Absolute instability in axisymmetric wakes: compressible and density variation effects, J. Fluid Mech. 600 (2008) 373-401.

[27] M. Abid, M. Brachet, P. Huerre, Linear hydrodynamic stability of circular jets with thin shear layers, Eur. J. Mech. B Fluids 12 (1993) 683-693.

[28] J.-M. Chomaz, Global instabilities in spatially developing flows: non-normality and nonlinearity, Annu. Rev. Fluid Mech. 37 (2005) 357-392.

[29] J.-M. Chomaz, Transition to turbulence in open flows: what linear and fully nonlinear local and global theories tell us, Eur. J. Mech. B Fluids 23 (2004) 385-399.

[30] P. Huerre, Open shear flow instabilities, in: G. Batchelor, K. Moffatt, G. Worster (Eds.), Perspectives in Fluid Dynamics, Cambridge, 2000, pp. 159-229.

[31] R.J. Deissler, The convective nature of instability in plane Poiseuille flow, Phys. Fluids 30 (1987) 2303-2305.

[32] W. Coenen, A. Sevilla, A. Sánchez, Absolute instability of light jets emerging from circular injector tubes, Phys. Fluids 20 (2008) 074104. 